\title{
Como os indicadores sociais e ambientais influenciam as taxas de suicídio - uma
}

\section{análise estatística}

\author{
How social and environmental indicators influence suicide rates - a statistical analysis \\ Cómo los indicadores sociales y ambientales influyen en las tasas de suicidio: un análisis estadístico
}

Recebido: 28/09/2021 | Revisado: 01/10/2021 | Aceito: 05/10/2021 | Publicado: 25/10/2021

Maurielle de Castro Ferreira

ORCID: https://orcid.org/0000-0001-5692-0103 Fundação Escola de Saúde Pública de Palmas, Brasil E-mail: mauriellecf@gmail.com

Maguylene Rosa da Silva

ORCID: https://orcid.org/0000-0001-6614-8743 Fundação Escola de Saúde Pública de Palmas, Brasil

E-mail: maguylenerosa@gmail.com

Daniela Aparecida Araújo Fernandes

ORCID: https://orcid.org/0000-0002-9834-0841

Universidade Federal do Tocantins, Brasil

Fundação Escola de Saúde Pública de Palmas, Brasil

E-mail: danimanduca@gmail.com

Angélica Jaconi

ORCID: https://orcid.org/0000-0002-9917-7767

Universidade Federal do Tocantins, Brasil

E-mail: angelicajaconi@yahoo.com.br

Magale Karine Rambo

ORCID: https://orcid.org/0000-0003-2529-9574

Universidade Federal do Tocantins, Brasil

E-mail: magalerambo@uft.edu.br

\begin{abstract}
Resumo
Uma análise multivariada de dados, avaliando os parâmetros sociais e ambientais do estado do Tocantins, mais especificamente da capital, Palmas, foi utilizada como forma de entender as causas das violências autoprovocadas - os suicídios. Os resultados estatísticos apresentaram forte relação entre as variáveis faixa etária, ocupação e sexo, enquanto que a variável escolaridade não apresentou grande influência. Já as variáveis ocupação e faixa etária, com $20,7 \%$ da variância, estão altamente correlacionadas. As variáveis escolaridade, raça/cor, tipo de óbito e local da ocorrência não apresentaram distinção entre as amostras. É importante destacar, que para mudança da realidade de saúde se faz necessário fortalecer as Políticas de Redução da Morbimortalidade por Acidentes e Violências e de Promoção de Saúde a fim de promover equidade e a melhoria das condições dos modos de viver, potencializando a saúde e reduzindo vulnerabilidades e riscos decorrentes dos determinantes sociais, econômicos, políticos e ambientais.
\end{abstract}

Palavras-chave: Tocantins; Análise por componentes principais; Violência.

\begin{abstract}
A multivariate data analysis, evaluating the social and environmental parameters of the state of Tocantins, more specifically the capital, Palmas, was used as a way to understand the causes of self-inflicted violence - suicides. The statistical results showed a strong relationship between the variables age, occupation and sex, while the variable education did not have a great influence. The variables occupation and age group, on the other hand, with $20.7 \%$ of the variance, are highly correlated. The variables education, race/color, type of death and place of occurrence did not show any distinction between the samples. It is important to highlight that, in order to change the health reality, it is necessary to strengthen the Policies for the Reduction of Morbimortality from Accidents and Violence and for the Promotion of Health in order to promote equity and improve the conditions of the ways of living, enhancing health and reducing vulnerabilities and risks arising from social, economic, political and environmental determinants.
\end{abstract}

Keywords: Tocantins; Principal component analysis; Violence.

\section{Resumen}

Se utilizó un análisis de datos multivariante, que evaluó los parámetros sociales y ambientales del estado de Tocantins, más específicamente la capital, Palmas, como una forma de comprender las causas de la violencia autoinfligida: los suicidios. Los resultados estadísticos mostraron una fuerte relación entre las variables edad, ocupación y sexo, mientras 
que la variable educación no tuvo una gran influencia. Las variables ocupación y grupo de edad, en cambio, con el 20,7\% de la varianza, están altamente correlacionadas. Las variables educación, raza / color, tipo de muerte y lugar de ocurrencia no mostraron distinción entre las muestras. Es importante resaltar que, para cambiar la realidad de la salud, es necesario fortalecer las Políticas para la Reducción de la Morbimortalidad por Accidentes y Violencia y para la Promoción de la Salud con el fin de promover la equidad y mejorar las condiciones de las formas de vivir, mejorar la salud y reducir las vulnerabilidades y los riesgos derivados de los determinantes sociales, económicos, políticos y ambientales.

Palabras clave: Tocantins; Análisis de componentes principales; Violência.

\section{Introdução}

A saúde pública tem sido constantemente afetada pelas taxas de morbimortalidade. No Brasil, o suicídio foi a segunda principal causa de morte entre jovens de 15 a 29 anos, estando atrás apenas dos acidentes de trânsito (Organização Pan-Americana de Saúde [OPAS], 2019). Cerca de 1 milhão de óbitos, ou 1,4\%, são decorrentes do suicídio (World Health Organization [WHO], 2014). No Brasil, entre 2011 a 2015 foram registrados 55.649 óbitos por suicídio, com uma taxa geral de 5,5/100 mil habitantes, já no período de 2011 o número correspondeu a 5,3 e variou com 5,7 em 2015, fato esse de grande magnitude que deve ser enfrentado (Brasil, 2017).

Dentre as causas externas - as violências autoprovocadas - os suicídios, assim como, as tentativas, têm gerado grande investimento para os sistemas de proteção social e cuidados com a saúde, provocando elevados gastos aos cofres públicos. Os gastos na saúde, como por exemplo, com procedimentos hospitalares, internações e tratamentos, assim como, na seguridade social aumentou o pagamento de pensões e aposentadorias por mortes ou invalidez (Ferreira, Pessoa, Barros, Figueiredo \& Minayo, 2014).

Apesar dos gastos com cuidados de atenção à saúde e dos custos econômicos em relação a perda de vida produtiva por morte, incapacidade ou prisão, ainda há poucos estudos para avaliar o impacto econômico dessas causas (WHO, 2014).

As causas externas por décadas permanecem se destacando no cenário epidemiológico do Brasil e do mundo (Brasil, 2015). Os acidentes e violências têm determinado um importante impacto na saúde das populações em vários países (Secretaria de Estado da Saúde de São Paulo, 2006). O cenário epidemiológico das causas externas na sociedade capitalista impacta a realidade social da população, bem como interfere na forma de produzir saúde.

O cenário nacional de mortalidade por suicídio apontou sobre a necessidade do uso de ferramentas de controle e fiscalização, para orientação dos serviços de saúde. Os indicadores sociais contribuem com o trabalho de vigilância em saúde, bem como possibilitam a identificação das desigualdades sociais relacionadas às condições de pobreza e falta do acesso a serviços, programas, projetos e benefícios sociais (Instituto Brasileiro de Geografia e Estatísticas [IBGE], 2019). Esses indicadores podem auxiliar os profissionais de saúde na construção do perfil epidemiológico e mapeamento dos territórios que apresentem maior risco e vulnerabilidade social.

Assim, foi utilizada a fonte de dados secundários para validar a existência de evidências, que comprovem a eficácia dos indicadores sociais enquanto estratégia de trabalho. Portanto, o Instituto Brasileiro de Geografia e Estatística (IBGE), órgão da administração pública federal, vinculado ao Ministério da Economia, realiza, bem como, disponibiliza periodicamente estudos e análises estatísticas relacionadas ao território, população, economia e condições de vida da população. Dessa forma, os dados referentes à educação, saneamento básico e renda que se encontram descritos nesta pesquisa são resultantes da extração dos dados disponibilizados pelo IBGE no período de análise deste estudo.

Nesse sentido, a variável educação constitui-se em um importante indicador social que possibilita a verificação de outros aspectos sociais que podem implicar de forma direta ou indireta na condição social do indivíduo, como o nível de escolaridade, 
a taxa de analfabetismo, as desigualdades no acesso à educação, a frequência escolar, entre outros fatores (Guzzo \& Euzébios Filho, 2005).

O saneamento básico é outra variável de suma importância e que possui incidência direta na qualidade de vida da população, pois, o acesso precário ou a falta aos meios adequados de saneamento corrobora para o aparecimento de inúmeras doenças na população, bem como, na degradação do meio ambiente.

Através da distribuição de renda da população em geral é possível realizar análises das desigualdades regionais, bem como, no estudo de outros fatores sociais, econômicos e de saúde que interferem ativamente nas condições de vida dos indivíduos.

Nesta perspectiva, este estudo apresenta o perfil de mortalidade por suicídio no município de Palmas, associados a reflexão dos indicadores sociais. Descreve as condições sociodemográficas, a evolução dos óbitos no período listado, bem como a taxa bruta de mortalidade. A fim de estimular a produção de estudos epidemiológicos e estatísticos com base na realidade social de determinada situação de saúde, esta pesquisa apresenta relevância para a saúde coletiva, ao identificar vulnerabilidades sociais e necessidades de saúde, tendo em vista, mudanças dos processos de trabalho e das práticas de prevenção e promoção da saúde, e mais especificamente sobre o enfrentamento do suicídio.

Importante destacar que se faz necessário o fortalecimento das políticas públicas, a fim de promover equidade e a melhoria das condições e dos modos de viver, potencializando a saúde e reduzindo vulnerabilidades e riscos decorrentes dos determinantes sociais, econômicos, políticos, culturais e ambientais (Brasil, 2014).

\section{Metodologia}

A pesquisa quantitativa atua sobre um problema humano ou social. Esta área do conhecimento busca respaldo em uma teoria de teste com variáveis quantificadas e analisadas pelo método estatístico, pois possui o objetivo de determinar a possibilidade de generalizações previstas na teoria (Knechtel, 2014).

Esta pesquisa apresenta relevância de estudo epidemiológico, com abordagem descritiva do método quantitativo. Foram utilizadas fontes de dados secundárias do Sistema de Informação de Mortalidade (SIM), prontuário eletrônico do Sistema e-SUS Atenção Primária (e-SUS) e Instituto Brasileiro de Geografia e Estatística (IBGE).

O período de estudo nos anos de 2017 a 2019, obteve uma amostra de 58 óbitos por residentes em Palmas. O período estabelecido compreende a data de implantação do e-SUS, no município de Palmas, sendo esta a ferramenta que supostamente haveria informações qualitativas dos indicadores sociais.

Para a coleta de dados, foi utilizado ainda, um roteiro baseado na declaração de óbito, na qual, foram filtrados os dados no SIM e no e-SUS, com o objetivo de analisar relatos dos profissionais de saúde a respeito do perfil social da população que veio a óbito por suicídio. Dessa forma, para a construção do perfil epidemiológico e sua relação com os indicadores sociais, as variáveis analisadas foram: sexo; faixa etária; raça/cor; gênero; escolaridade; deficiência/transtornos; situação conjugal; ocupação; renda; benefícios; habitação; condição de moradia; saneamento/água; esgoto; lixo; energia elétrica; acesso aos dispositivos de saúde/assistência; fonte de informação e local de ocorrência do óbito.

A coleta dos dados pelo sistema de prontuário do e-SUS teve como objetivo na identificação de informações inerentes às condições sociodemográficas da amostra da pesquisa. As informações coletadas foram disponibilizadas por meio de planilha excel para a análise dos dados, sendo transformado em forma de porcentagem o resultado obtido. A consideração das variáveis educação, saneamento e renda foram subsidiadas pelas pesquisas realizadas periodicamente pelo IBGE e utilizadas para o conhecimento acerca desses indicadores sociais nos anos equivalentes aos dados da pesquisa. 
Os dados após estratificados no sistema de informação do SIM foram tabulados pelo programa Tabwin versão 4.1, a partir de gráficos e tabelas. Os resultados representam série histórica da evolução dos óbitos de suicídio no período listado. O cálculo da taxa bruta de mortalidade desses óbitos ocorridos no período de um ano, foi calculado por cem mil habitantes em relação à estimativa anual da população total do município segundo o IBGE. Já, a causa básica do óbito está em conformidade com a Classificação Internacional de Doenças (CID-10), publicada pela Organização Mundial de Saúde, a partir de informações padronizadas sobre a codificação de doenças relacionadas à saúde.

Foi utilizado o método de análise de componentes principais (PCA) e recurso do software R (R Core Team, 2020) com o pacote Stats. A matriz de dados apresentou dimensões 58 x 8, para análise de 58 amostras por 8 variáveis. Os dados foram previamente auto escalados e centrados, antes de serem submetidos à análise de componentes principais, uma vez que há uma grande variação de respostas das diversas variáveis, ou seja, diferem em ordem de grandeza.

Essa pesquisa foi submetida ao Comitê de Ética e Pesquisa com aprovação pelo número do CAAE: 32014620.10000.9187 e parecer de número: 4.060.282. Todos os procedimentos desenvolvidos na pesquisa encontram-se em conformidade com o CNS nº 466/12 e suas complementares, bem como, a CONEP nº01/13, especialmente no que se refere à integridade e proteção dos participantes na pesquisa.

\section{Resultados e Discussão}

Com relação aos coeficientes de óbitos de suicídio da população de Palmas (TO), em 2017 houveram 14 óbitos, e coeficiente de 4,9 por 100 mil habitantes, em 2018 houveram 24 óbitos e coeficiente de 8,0 por 100 mil habitantes, em 2019 foram 20 óbitos e coeficiente de 6,5 por 100 mil habitantes. Pode-se observar uma tendência crescente de suicídios de 2017 a 2018 de $71 \%$ e um decréscimo de 2018 a 2019 de $17 \%$.

Na Figura 1, as componentes CP1 e CP2 descrevem 38,2\% da variação total dos dados e fornecem informações discriminatórias das amostras. A primeira componente principal (CP1) descreve apenas 20,7\% da variação total e a segunda (CP2) 17,5\%. Foi possível constatar a importância de cada variável neste conjunto de dados, pois explica 100\% da variância na oitava dimensão. Ainda na Figura 1, se observa a influência das variáveis sobre as amostras. Constata-se que as variáveis faixa etária, ocupação e sexo tem uma maior influência das amostras em 38,2\% de variância, por outro lado, a variável escolaridade não tem grande influência. 
Figura 1. (a) Porcentagem da variância e número de componentes principais (b) Influência das variáveis sobre as amostras.
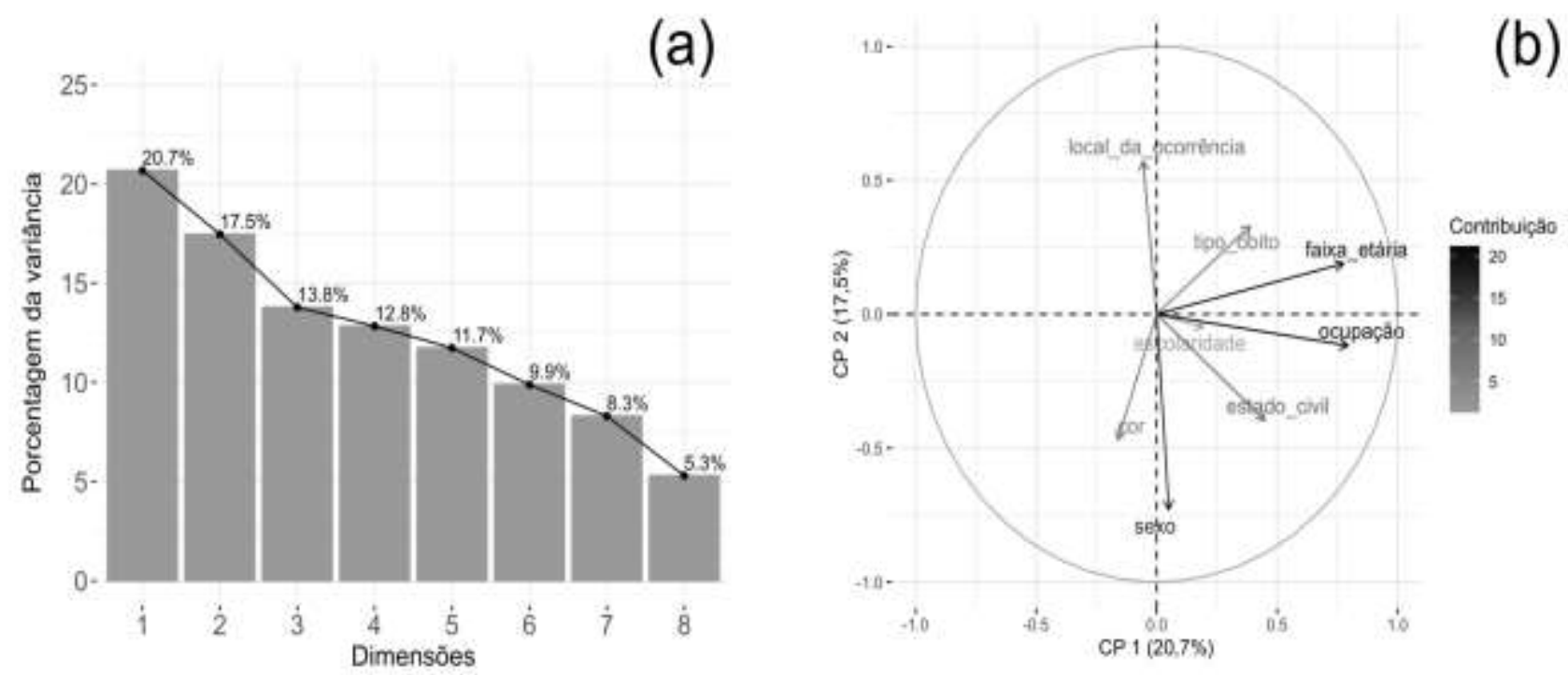

Fonte: Autores (2021).

A Figura 2 analisa variavéis de ocupação, faixa etária e sexo, sendo possível observar a discriminação entre grupos, ou seja, com um comportamento distinto pelas amostras classificadas. Com base nisso, a ocupação e faixa etária, a CP1 (20,7\%) é responsável pela distinção das variáveis, por sua vez essas são altamente correlacionadas. Por outro lado, na figura (c) sexo a CP2 $(17,5 \%)$ explica a distinção entre o sexo. 
Figura 2. Análise por componentes principais da variável (a) ocupação; (b) faixa etária, (c) sexo.
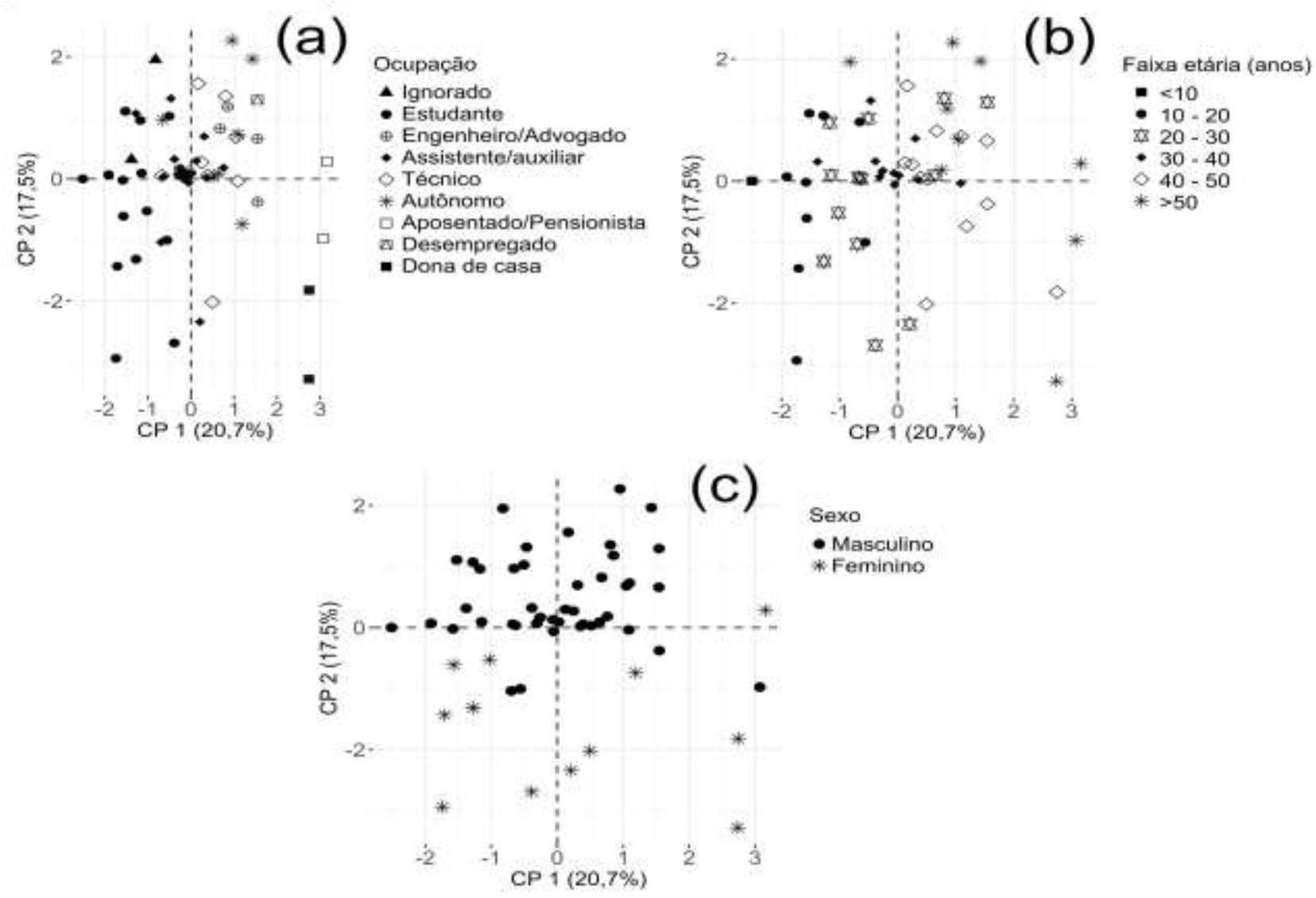

Fonte: Autores (2021).

Conforme apresenta a Figura 3, as variáveis estudadas como por exemplo, escolaridade, raça/cor, tipo de óbito e local da ocorrência não apresentaram distinção entre as amostras.

Dos óbitos registrados, 22,4\% representam o sexo feminino e 77,5\% são masculinos. Na faixa etária, a maior frequência de suicídio ocorreu nas faixas de 21-30 anos com 24,1\%, seguidos de 31-40 anos, 18,9\%, diminuído os níveis a partir dos 41-50 anos, 20,6\%, 51-60 anos, 6,8\% e apenas $>61$ anos apresentou $10,3 \%$.

Já, a raça/cor mais representativa são os pardos com 65,5\% e brancos com 27,5\%. Não foi registrado nenhum óbito em pessoas com raça/cor amarela e indígena, e apenas $1,7 \%$ são da cor preta.

Em relação à variável escolaridade, as taxas variam sendo o ensino fundamental I de 1,7\%, fundamental II 10,3\%, médio representam $15,5 \%$, superior completo são $24,1 \%$, nível superior incompleto apenas $17,2 \%$ e $31,0 \%$ não especificados. 
Figura 3. Análise de componentes principais (PCA) discriminando as variáveis e amostras tais como (a) escolaridade; (b) cor; (c) tipo de óbito e (d) local da ocorrência.
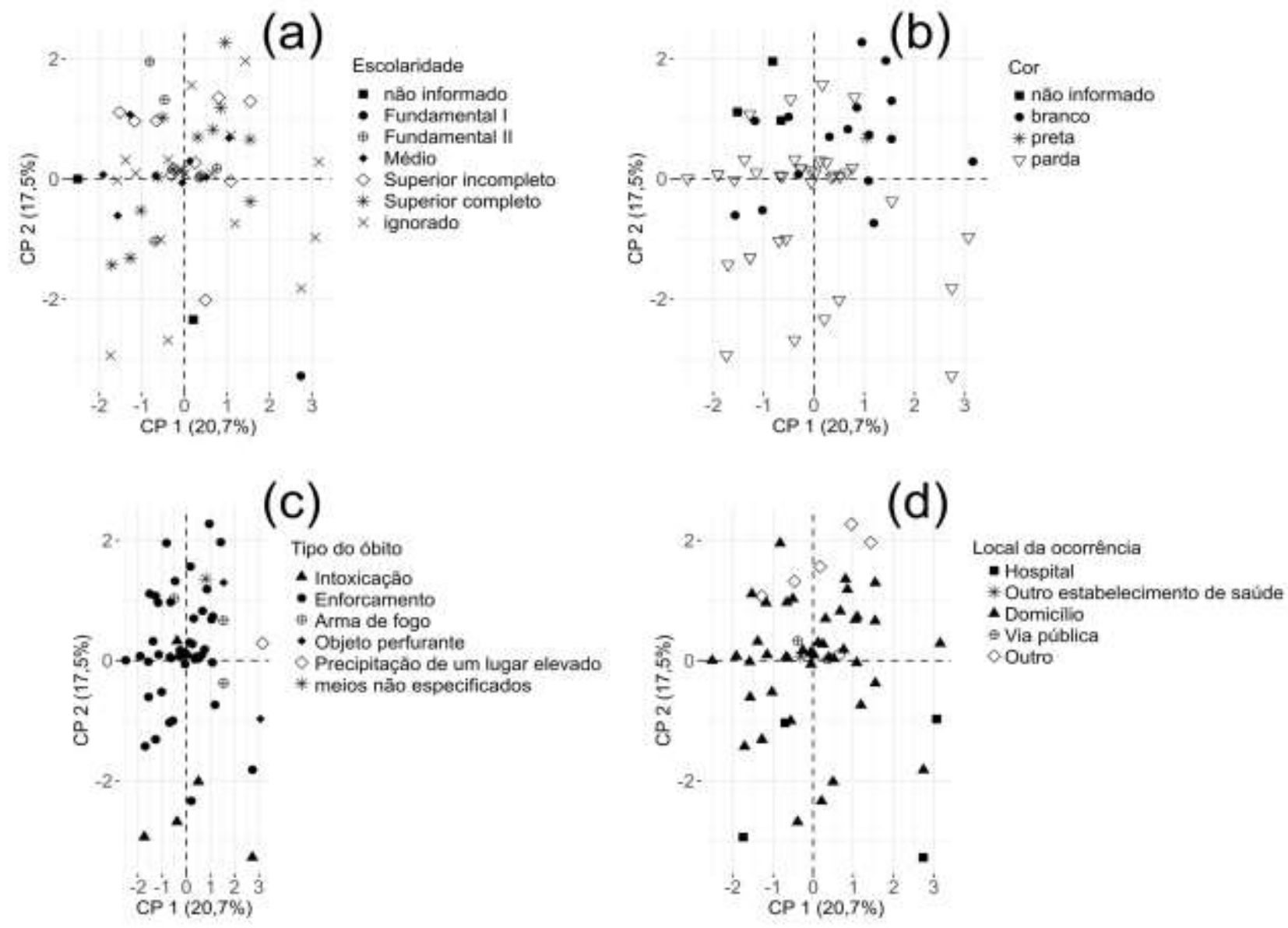

Fonte: Autores (2021).

A respeito dos demais dados observados, a situação conjugal é descrita em sua maioria pelos solteiros que chegam a $55,1 \%$, aqueles que são casados ou com união consensual apontam 22,4\%, os de união estável com 10,3\% e os separados 5,1\%.

A ocupação corresponde a 27,5\% sendo de estudantes, motorista de carro de passeio 8,6\% e representante comercial autônomo 6,8\%. Já a grande maioria das categorias profissionais, a exemplo, comerciante varejista, advogado, servente de obras, agente de segurança, gerente administrativo e aposentado/pensionista, bem como os ignorados, totalizaram 3,4\% cada. As demais ocupações não apresentaram significância.

Sobre a deficiência/transtorno consta que $15,5 \%$ possui algum transtorno mental, enquanto que em 5,1\% apresentaram outros transtornos/deficiência e, 56,8\% não especificados. Não se teve acesso ao E-SUS de 22,4\% dos pesquisados por não estarem cadastrados na base de dados do município ou não terem ainda o registro no sistema.

A causa básica de maior destaque de óbito é o CID X.70 (lesão autoprovocada intencionalmente por enforcamento, estrangulamento e sufocação) com 77,5\%, seguido da causa X.69 (autointoxicação por exposição, intencional, a outros produtos químicos e substâncias nocivas não especificadas) de 6,8\%. A causa X.74 (lesão autoprovocada intencionalmente por disparo de outra arma de fogo e de arma de fogo não especificada), contou com 5,1\%. E o X.78 (lesão autoprovocada intencionalmente por objeto cortante ou penetrante - local não especificado), resultando em 3,4\%. A autointoxicação são subclassificadas pelo tipo de substância, e somando estas causas chama a atenção a sua prevalência com 10,2\%. 
A educação é um indicador social com impacto expressivo na vida dos sujeitos, pois, ter acesso aos meios educacionais (escola, ensino) corrobora para o desenvolvimento social, político, cultural e econômico do indivíduo, concebe sujeitos críticos e sobretudo, resultando na diminuição das desigualdades sociais (Cury, 2002). Conforme preconizado na Constituição Federal, a educação, direito de todos e dever do Estado e da família, será promovida e incentivada com a colaboração da sociedade, visando ao pleno desenvolvimento da pessoa, seu preparo para o exercício da cidadania e sua qualificação para o trabalho (Brasil, 1998).

As taxas de escolarização no Brasil nos anos alusivos da pesquisa corresponderam a 99,2\%, 99,3\% e 99,7\% respectivamente, enquanto que a taxa de analfabetismo na população de 15 anos ou mais de idade correspondeu a $6,9 \%, 6,8 \%$ e $6,6 \%$ respectivamente (IBGE, 2020). Conforme os dados apresentados, houve um tímido aumento entre os anos de 2107 e 2108 da taxa de escolarização em relação ao ano de 2019 que apresentou um crescimento de 0,4\% comparado ao ano anterior. Da mesma forma ocorreu com a taxa de analfabetismo de pessoas com 15 anos ou mais de idade, no qual demonstra uma queda em torno de $4 \%$ entre os anos referidos.

O saneamento básico consiste num fator que influencia diretamente nas condições da promoção de saúde e de vida da população. Para, além disso, ter acesso a um esgotamento precário e inadequado provoca danos e a degradação de bacias hidrográficas, a biodiversidade dos rios, dos mananciais e do solo (Ferreira \& Garcia, 2017).

O acesso ao abastecimento de água por rede geral, esgotamento sanitário por rede coletora ou pluvial e a coleta regular do lixo urbano colaboram principalmente na prevenção de várias doenças causadas por vírus ou bactérias como as infecções gastrointestinais, doenças dermatológicas, hepatites, cólera, entre outras.

As regiões norte e nordeste do país são as que mais sofrem restrições dos serviços de saneamento e fornecimento de água por rede segura. Conforme o Sistema Nacional de Informações sobre Saneamento (SNIS), em 2019 a região norte apresentou 70,4\% da população urbana com acesso à rede de água, 15,8\% atendidas com rede de esgoto e 97,5\% com coleta de resíduos sólidos (Brasil, 2019). De acordo com dados de 2010, entre 2000 a 2008 houve uma redução no número de municípios sem implantação de rede coletora de esgoto no estado do Tocantins, o que contribuiu para a eliminação de valas e esgoto a céu aberto no período (Lima, 2019).

Quanto ao município de Palmas (TO), estudos do Instituto Trata Brasil (2020) revelam que cerca de 85\% da população local recebe o serviço de coleta de esgoto e 66,9\% desse volume de esgoto é tratado. De acordo com o mesmo estudo, a cidade ocupa o $28^{\circ}$ lugar ficando atrás apenas de quatro capitais brasileiras no ranking das 100 maiores cidades com saneamento básico do país.

No indicador social - renda -as regiões sul, sudeste e centro-oeste possuem rendimento médio aproximado entre si e superior às regiões norte e nordeste, sendo o fator que justifica as regiões com menor rendimento o elevado percentual de domicílios com rendimento per capita de até 1⁄2 salário-mínimo. Nas regiões norte e nordeste apenas 7,8\% e 7,7\% da população, respectivamente, tinham o rendimento domiciliar per capita superior a dois salários-mínimos, sendo que nas demais regiões esses percentuais eram de $20 \%$ ou mais, ficando acima da média nacional que era de 16,2\% (IBGE, 2018).

Importante ressaltar que aspectos como geográficos, raça e cor influenciam diretamente nos rendimentos entre os grupos de pessoas e domicílios. A origem dos rendimentos da população de uma determinada localidade é um fator relevante na identificação das diferenças regionais apresentadas. No Brasil, 73,8\% dos rendimentos provêm do trabalho, 19,4\% de aposentadoria e pensão, e 6,9\% de outras fontes (IBGE, 2018).

Em 2018 o salário médio dos palmenses correspondia a 3.9 salários-mínimos e a proporção de pessoas ocupadas era de 48,3\%. Em relação aos demais municípios do estado, Palmas ocupava a posição 01 de 139 municípios e 01 de 139 , 
respectivamente, enquanto que na comparação com as demais cidades do país a posição 36 de 5570 municípios e 64 de 5570, respectivamente (Brasil, 2014).

\section{Considerações Finais}

No município de Palmas houve um aumento no acesso da população ao saneamento básico nos anos referentes da pesquisa, isso requer dizer, maior acesso na distribuição de água tratada, coleta de esgoto por rede coletora e da coleta do lixo doméstico; da mesma forma, houve também uma maior inclusão de alunos aos anos iniciais da educação básica. Em relação a renda, percebe-se que os palmenses possuem salários de acordo com a média nacional e, lideram o ranking quando comparado aos demais municípios do Tocantins. Observa-se que os dados apresentados são positivos e indicam melhora e aumento da qualidade de vida da população local.

Apesar de não inferir a relação do perfil da mortalidade com os indicadores sociais, devido à incompletude das informações, nos bancos de dados, os indicadores sociais são ferramentas úteis na vigilância da saúde, na intenção de contribuir com o monitoramento, a avaliação e a qualidade dos serviços públicos do Sistema Único de Saúde. Mesmo com tantos desafios para se produzir saúde, os indicadores sociais podem contribuir com a redução da mortalidade por suicidio, considerando também que "os registros de tentativas de suicido em Palmas estão acima da média nacional o que sugere a necessidade de estratégias de promoção/prevenção e intervenção para redução da morbimortalidade" (Fernandes, Ferreira \& Castro, 2017, p.9).

A partir de estudos que comprovam a eficácia dos indicadores sociais no auxílio de gestores e profissionais da área de saúde a desenvolverem ações, projetos e programas voltados para as políticas públicas. Este estudo sugere a sua utilização nas ações de prevenção e promoção de saúde. Através da avaliação periódica dos indicadores será possível obter melhores resultados da situação dos territórios de saúde, como possibilitará qualidade na gestão dos sistemas de informação e contribuirá com a identificação de agravos e determinantes que resultam de processos produtivos, econômicos e de trabalho.

Não obstante, as questões de ordem socioeconômicas que geram o estigma social, o preconceito e as questões de cunho jurídico reforçam a invisibilidade das violências autoprovocadas. Dessa forma, propomos, com base em evidências e com intervenções de baixo custo, a realização de atividades de educação permanente envolvendo os profissionais da atenção primária. Afim do preenchimento qualitativo do E-sus, com intuito do aprimoramento do referido sistema e no desenvolvimento de estratégias que melhor atendam às demandas sociais dos indivíduos. Pretende-se mudanças significativas nos modelos de atenção e práticas dos processos de trabalho, bem como integrar grupos de risco e pessoas em situação de vulnerabilidade social.

\section{Agradecimentos}

Os autores agradecem à Universidade Federal do Tocantins, e ao PPGCiamb (Programa de PósGraduação em Ciências Ambientais) pelo apoio a publicação.

\section{Referências}

Brasil. (1988). Constituição da República Federativa do Brasil de 1998. Senado Federal; 1988.https://www.senado.leg.br/ativ idade/const/con1988/CON1988_05.10.1988/art_205_.asp

Brasil. (2014). Ministério da Saúde. Secretaria de Vigilância à Saúde. Secretaria de Atenção à Saúde. Política Nacional de Promoção da Saúde: PNaPS: revisão da Portaria MS/GM no 687, de 30 de março de 2006.http://bvsms.saude.gov.br/bvs/publicacoes/politica_nacional_promocao_saude_pnaps.pdf

Brasil. (2015). Ministério da Saúde. Secretaria de Vigilância em Saúde, Departamento de Vigilância de Doenças e Agravos Não Transmissíveis e Promoção da Saúde. Saúde Brasil 2014: uma análise da situação de saúde e das causas externas. http://svs.aids.gov.br/dantps/centrais-de-conteudos/publicacoes/saudebrasil/saude-brasil-2014-uma-analise-da-situacao-de-saude-e-das-causas-externas.pdf 
Brasil. (2017). Ministério da Saúde. Secretaria de Vigilância em Saúde. Boletim Epidemiológico. Suicídio. Saber, agir e prevenir, 48(30). https://portalarquivos2.saude.gov.br/images/pdf/2017/setembro/21/2017-025-Perfil-epidemiologico-das-tentativas-e-obitos-por-suicidio-no-Brasil-e-a-rede-deaten--ao-a-sa--de.pdf

Brasil. (2019). Ministério do Desenvolvimento Regional. SNIS, Sistema Nacional de Informações sobre Saneamento. http://snis.gov.br/painelinformacoessaneamento-brasil/web/painel-setor-saneamento

Cury, C. R. J. (2002). Direito à educação: direito à igualdade, direito à diferença. Cadernos de pesquisa, 119(01), 245-262. https://www.scielo.br/pdf/cp/n116/14405.pdf

Ferreira, N. S., Pessoa, V. F., Barros, R. B., Figueiredo, A. E. B., \& Minayo, M. C. S. (2014). Fatores de risco relacionados com suicídios em Palmas (TO), Brasil, 2006-2009, investigados por meio de autópsia psicossocial. Ciência \& Saúde Coletiva, 19(01), 115-126. https://doi.org/10.1590/1413-81232014191.222

Ferreira, M. P., \& Garcia, M. S. D. (2017). Saneamento básico: meio ambiente e dignidade humana. Dignidade Re-Vista, 2(3),1-12.http://periodicos.pucrio.br/index.php/dignidaderevista/article/view/393

Fernandes, D. A. A., Ferreira, N. S. \& Castro, J. G. D. (2017). Perfil Epidemiológico das tentativas de suicídio em Palmas-Tocantins, de 2010 a 2014. TempusActas de Saúde Coletiva, 10(4) 09-23. https://doi.org/10.18569/tempus.v11i1.2016

Guzzo, R. S. L., \& Euzébios, F. A. (2005). Desigualdade social e sistema educacional brasileiro: a urgência da educação emancipadora. Escritos sobre Educação, 4 (2), 39-48. http://pepsic.bvsalud.org/scielo.php?script=sci_arttext\&pid=S1677-98432005000200005\&lng=pt\&tlng=pt

IBGE. (2018). Instituto Brasileiro de Geografia e Estatísticas. Síntese de indicadores sociais: uma análise das condições de vida da população brasileira.

R Development Core Team. 2019: R: A language and environment for statistical computing. R Foundation for Statistical Computing, Vienna, https://www.rproject.org/.https://sistemas.uft.edu.br/periodicos/index.php/csdt/issue/view/348/293

OPAS. (2019). Organização Pan-Americana da Saúde, Organização Mundial de Saúde, OMS. https://www.paho.org/bra/index.php?o ption=com_content\&view=article\&id=6017:suicidio-uma-pessoa-morre-a-cada-40-segundos-afirma-oms \&Itemid=839

Secretaria de Estado da Saúde de São Paulo. (2006). O impacto dos acidentes e violências nos gastos da saúde. Revista de Saúde Pública, 40(3), 553-556. https://www.scielo.br/pdf/rsp/v40n3/28.pdf

WHO. (2014). World Health Organization. Country reports and charts available; 2014. https://www.who. int/mental_ health/ prevention/sui cid e/country _rep orts/en/ index

WHO. (2014). World Health Organization. Preventing suicide: a global imperative. WHO; 2014. https://www.who.int/publications/i/item/preventing-suicide-aglobal-imperative 